\title{
Physician-scientists in the pandemic era: tidal wave or rising tide?
}

\author{
Lorraine B. Ware
}

Departments of Medicine and Pathology, Microbiology and Immunology, Vanderbilt University School of Medicine, Nashville, Tennessee, USA.

A s we gather for our annual meeting virtually for the first time in the history of the American Society for Clinical Investigation (ASCI), it is an honor to address you as the 111th president of this society. Just over a year ago, on March 11, 2020, as the global SARS-CoV-2 pandemic began to take hold, the leadership of the ASCI, the Association of American Physicians (AAP), and the American Physician Scientists Association (APSA) made the difficult decision to cancel the 2020 Joint Meeting. The annual ASCI gathering of clinical investigators has previously been canceled only twice in its 112-year history, in 1943 and 1945, and then only due to the major global disruption of World War II (1).

In March of 2020, many of us, including myself, were hoping that the COVID-19 pandemic would be short-lived and easily contained, a distraction, but not a major disruption. Instead, this pandemic has posed a serious threat to life and livelihood. For the majority of Americans alive today who have not lived through a major world war, the pandemic is the greatest disruption and biggest collective existential threat that we have ever faced. There are some encouraging signs in April 2021 as case numbers drop below January highs and vaccination increases. However, the rise of new variants of SARS-CoV-2 that are potentially more contagious and more virulent, coupled with concerns about vaccine hesitance and inadequate global access to vaccines, portends a future that is still clouded with uncertainty.

Preparing for this annual presidential address is a rite of passage for ASCI presidents, and it has been an enlightening and humbling task to review the speeches of many of my esteemed predecessors. In his 2002 address, Dr. David Ginsburg, the 92nd president of the ASCI, identified five recurring themes that have consistently appeared in prior ASCI presidential addresses (2), including the future of the physician-scientist, defining clinical investigation, ASCI "state of the union," research funding, and education and mentoring. All of these topics continue to be important for the ASCI today. I will focus primarily on the impact of the SARS-CoV-2 pandemic on two of these themes, the future of the physician-scientist and the changing landscape of clinical investigation.

The pandemic has had both positive and negative effects on the physicianscientist community. One positive impact has been to underscore the central role that a vibrant community of well-trained physician-scientists plays in advancing biomedical science and public health. Around the world, physician-scientists have been integral to every aspect of the scientific response to COVID-19. Earlier today, the AAP and ASCI honored one physician-scientist and ASCI member for his role in spearheading the US response to this pandemic, Dr. Anthony Fauci; it has been heartwarming to see a physicianscientist gain such widespread recognition and respect from so many in the lay community. Many other physician-scientists, too numerous to name, have played pivotal roles in efforts to understand, track, treat, and contain the pandemic, including many in this audience. Physician-scientists have been at the forefront of studies of the fundamental biology of coronaviruses and in the development and testing of the novel mRNA and other vaccines that have shown unprecedented efficacy against this virus. The fact that widespread vaccination is taking place barely more than a year after this novel pandemic was recognized is breathtaking and illustrative of the acceleration of the pace of scientific discovery in the 21st century.

The pandemic has also highlighted the need for a physician-scientist community with diverse skills that extend beyond traditional bench investigation of pathophysiologic mechanisms of disease. As a critical care physician, I have seen firsthand the importance of physician-scientists with expertise in clinical trial design as new trials for agents to treat SARS-CoV-2 infection have been implemented at previously unthinkable speeds. Novel clinical trial designs, including platform and pragmatic trials, have bolstered our armamentarium with new therapies, including remdesivir and monoclonal antibodies, and repurposed therapies, such as dexamethasone and tocilizumab, and allowed us to rapidly discard ineffective therapies, such as hydroxychloroquine. Physician-scientists have also grappled at the bedside with other pandemic-related questions, such as what is the physiological impact of ventilating two patients with one ventilator, a question born of sheer desperation (3).

The pandemic has also highlighted the changing landscape of clinical investigation. The ASCI was founded in 1908, at a time when the exciting "new science" of physiology was replacing clinical-pathologic correlation at the frontier of biomedical discovery (1). This new science was largely the purview of a new breed of physician-investigators who embraced this fledgling field and eventually established a new journal in 1924 to publish their findings, the Journal of Clinical Investigation. As a lifelong student of pulmonary physiology, it was fascinating to see the physiologic focus of articles published in the first issue of JCI in 1924 (Figure 1). Over the past 110 years, the field of clinical investigation has grown and morphed repeatedly. Many new scientific disciplines have emerged to enrich clinical investigation, including those related 


\section{CONTENTS}

\section{NUMBER 1, October, 1924}

Purposes in Medical Research. An Introduction to the Journal of Clinical Investigation. Alrred E. CoHn $\ldots \ldots \ldots \ldots \ldots \ldots \ldots \ldots \ldots, 1$

Studies on the Specific Gravity of the Urine. O. H. Perry Pepper....... 13 On the Relation Between Conductivity and Chlorides in the Urine. H. C.

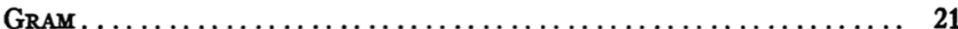

Blood Reaction and Blood Gases in Pneumonia. A. B. Hastings, J. M. Neml, H. J. Morgan AND C. A. L. BINGER ............... 25

A Method for the Determination of the Amount of Oxygen and Carbon Dioxide in the Mixed Venous Blood of Man. C. SIDNEY BuRwell AND G. Canby Robinson $\ldots \ldots \ldots \ldots \ldots \ldots \ldots \ldots \ldots \ldots \ldots \ldots \ldots \ldots \ldots \ldots$

The Rate of the Circulation of the Blood in Normal Resting Individuals. H. FIELD, JR., A. V. BOCK, E. F. GIIDEA AND F. L. LATHROP ......... 65

The Gaseous Content of the Blood and the Output of the Heart in Normal Resting Adults. C. Smeney BuRwell and G. Canby Robinson...... 87 Evidence that Digitalis Influences Contraction of the Heart in Man. ALFred E. Cohn and Harold J. Stewart...................... 97

Figure 1. Table of contents of the first issue of the Journal of Clinical Investigation, published in 1924.

to big data, precision medicine, and clinical trial design. Through the revitalized Institutional Representative program, the Council has made a concerted effort in the past five years to encourage nominations for ASCI membership of scientists from the entire spectrum of clinical investigation, including clinical, epidemiologic, and health services research. Compared to 2012 to 2016, in the past five years, there have been some encouraging trends in numbers of new members who are from fields other than internal medicine and from scientific disciplines other than bench investigation (Figure 2), and this remains a focus for the Council in the future. The JCI and JCI Insight also are aiming to publish more clinical medicine articles to better represent the breadth of scientific focus of our membership. Our journals have also responded nimbly to the challenge of the COVID-19 pandemic, altering editorial policies and publishing many key COVID-related manuscripts.

Despite bringing a rising tide of national and international attention to the key role that physician-scientists play in advancing the science of medicine, the pandemic has also overwhelmed physician-scientists with a tidal wave of tangible and intangible challenges. Lockdowns have shuttered research laboratories, and many laboratories remain closed or at reduced capacity. Non-COVID-related clinical research has been halted or limited at many academic centers and has been slow to resume. Shortages of key laboratory reagents and supplies have stalled important projects. Many physician-investigators and trainees have had to set aside research in order to shoulder increased clinical responsibilities during surges of patients with COVID-19. Despite this increased clinical effort, revenues at many academic centers have been heavily impacted and bridge funds to support investigators are less available. At home, many investigators have had to deal with loss of child care, elder care, and other important services, repeated quarantines due to exposures, school closures, and illness, and job losses among family and friends. These hardships have particularly impacted younger investigators, who in addition to being at a more vulnerable time in their scientific careers, are more likely to have young children at home; female and minoritized investigators are also particularly vulnerable.

Indeed, the intersection of the global pandemic with the \#MeToo movement that began in 2018 and the widespread demonstrations in 2020 in protest of police brutality and violence against Black and other minoritized communities has brought into sharp focus issues of systemic bias and inequities in science and health care (4). The COVID-19 pandemic has had a disproportionate impact on minoritized populations in the US, including Black, Hispanic, and Native American communities, with both higher infection rates and higher mortality. Yet these communities are largely unrepresented in the data that drive clinical care of the most severely ill
A

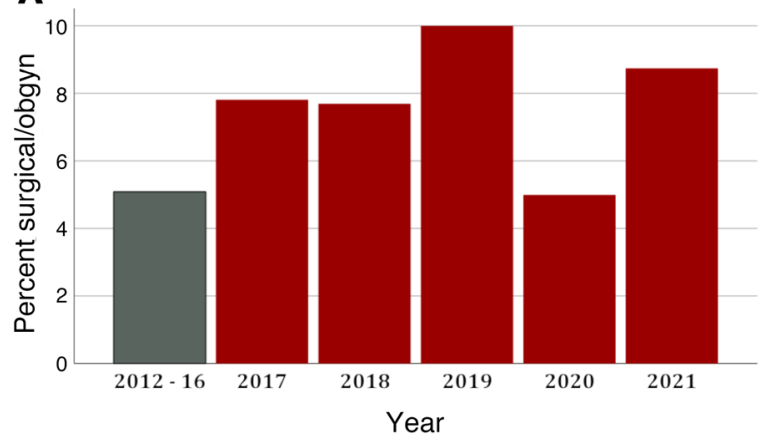

B

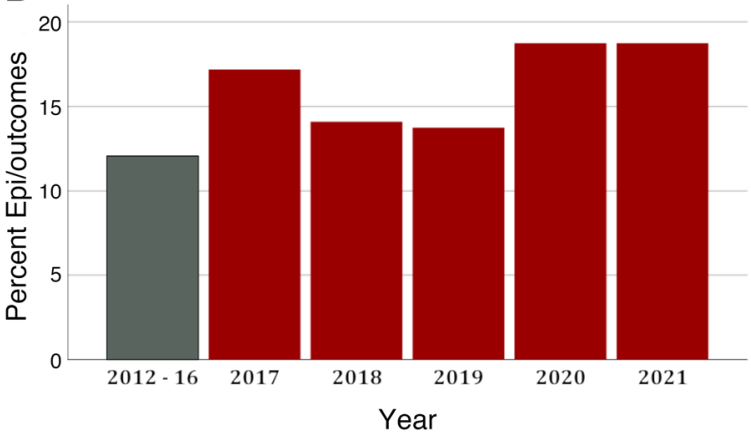

Figure 2. The representation of scientists from surgical fields, obstetrics and gynecology, epidemiology, and outcomes research among new members to the ASCI has increased in the past five years. Panels show percentages of newly elected members to ASCI by year from 2017 to 2021 compared with the five-year period between 2012 and 2016 for (A) new members from surgical fields and obstetrics and gynecology (Obgyn) and (B) new members from epidemiologic fields (Epi) and outcomes research. 
A

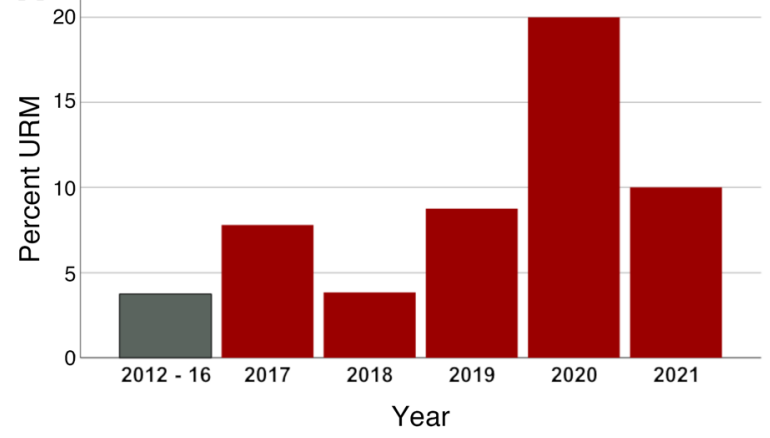

B

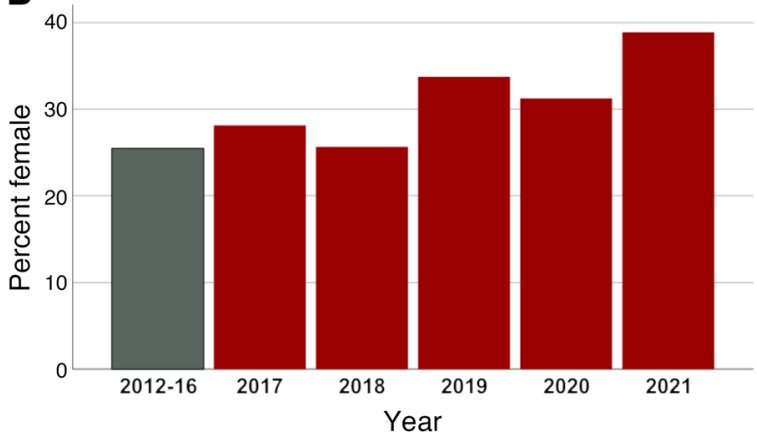

Figure 3. The representation of physician-scientists who are underrepresented in medicine or female among new members of the ASCI has increased in the past five years. Panels show percentages of newly elected members to ASCI by year from 2017 to 2021 compared with the five-year period between 2012 and 2016 for (A) new members from racial or ethnic groups that are underrepresented in medicine (URM) and (B) new members who are female.

(5), and these minoritized groups remain underrepresented in medicine and even more so in the ranks of physician-scientists. For example, Black people make up $13 \%$ of the US population but only $4 \%$ of US doctors and an even smaller percentage of physician-scientists (6). Yet it has been shown across a variety of fields, including science, that increased diversity feeds creativity and impact (7).

One of the first steps we undertook as an organization to address diversity and equity within the ASCI was to form a working group on diversity in 2018 with an aim of revising and modernizing our mission statement to be more inclusive; this task was completed in 2019 and the revised mission statement may be viewed on the ASCI website. In brief, the American Society for Clinical Investigation seeks to support the scientific efforts, educational needs, and clinical aspirations of physician-scientists to improve the health of all people. It is one thing to revise the wording of our mission, but an entirely different thing to actually be more inclusive; this is work that is in progress. As a female physician-scientist, I recall looking through the roster of members when I was first being nominated for ASCI membership many years ago and being struck by the low numbers of women and minorities and wondering if there would be a place for me in this society composed almost exclusively of White men. The Council has worked with our institutional representatives to increase the number of female and underrepresented minorities who are nominated for membership. This work is paying off (Figure 3) both in our membership and in our leadership where I am proud to have served as the eighth female president of the ASCI on a Council that was majority female and included the first Black editor of JCI, Dr. Rex Ahima, and the first female editor of one of our journals, JCI Insight editor Dr. Kathy Collins.

Despite these advances, we have much work to do. To be prepared for the next public health crisis, the rapid acceleration of biomedical science must be a rising tide that raises all boats, lifting and elevating the careers of female and minoritized physician-scientists along with the greater scientific community. In 2020, we amended the bylaws of the ASCI to form a permanent Diversity Equity and Inclusion Committee that is charged with ensuring that the organization, its programs, and its mentoring mechanisms promote and recognize diversity, inclusion, and equity. This committee is chaired by Councilor Dr. Sophie Paczesny. The first task that the committee is tackling is to develop definitions of groups that are underrepresented in medicine that can be used to survey our membership. Despite being an organization of scientists, it is striking that we have almost no data on the racial and ethnic composition of our membership and only recently started collecting these data as part of the nomination process. Other projects that the committee is undertaking include new mentoring programs and awards for female and underrepresented investigators. It is my hope that in the future, young investigators perusing the ranks of ASCI members as they contemplate their own nominations to ASCI will see more faces in this physician-scientist community that look like their own.
Finally, with regard to the "state of the union," I can assure you that despite the pandemic, the ASCI continues to thrive, both fiscally and organizationally. Although our society offices in Ann Arbor stand empty as remote work continues and our Council has not been able to gather in person, we have met virtually to conduct the business of our society. I am indebted to John Hawley, Executive Director, and Karen Guth, Managing Director, who have run the ASCI for over 20 years. Their collective institutional memory and steady hands at the helm have kept the ASCI on course despite the annual disruption of new leadership and the whims of each new council. I am also indebted to Dr. Kieren Marr and Dr. Kimryn Rathmell, remarkable leaders and role models who preceded me in this presidency and whose ideas and input shaped many of the active projects that the Council is working on currently. I leave you in the able hands of our incoming President, Dr. Hossein Ardehali, who has many exciting plans for the Society, along with President-Elect Dr. Sohail Tavazoie and our newly elected Vice President and outgoing Secretary-Treasurer Dr. Ben Humphreys. I would also like to welcome our three newly elected Council members, Drs. Julie Bastarache, Goutham Narla, and Elizabeth Speliotes.

In closing, thank you for the opportunity to have served as president during this challenging year. I will conclude by welcoming our new members. You are joining a society that, although steeped in tradition, was born of change. The ASCI will continue to adapt and evolve in response to the changing world and the changing landscape of clinical investigation. I encourage you to take 
advantage of the opportunities within this society to network, to publish in our journals, and to get involved in our committees, institutional representation, and leadership.

Address correspondence to: Lorraine B. Ware, T1218 MCN, 1161 21st Avenue S, Nashville, Tennessee 37232-2650, USA. Phone: 615.322.3412; Email: Lorraine. ware@vumc.org.
1. Howell JD. A history of the American Society for Clinical Investigation. J Clin Invest. 2009;119(4):682-697.

2. Ginsburg D. The history and evolution of the ASCI: déjà vu all over again. American Society for Clinical Investigation. JClin Invest. 2002;110(12):S1-S4.

3. Beitler JR, et al. Ventilator sharing during an acute shortage caused by the COVID-19 pandemic. Am J Respir Crit Care Med. 2020;202(4):600-604.

4. Ware LB, et al. A deliberate path toward diversity, equity, and inclusion within the ASCI. J Clin Invest. 2020;130(10): 5031-5032.

5. Saleem D, et al. Ethnic and sex representation in trials shaping best practice for COVID-19. Ann Am Thorac Soc. 2021;18(2):371-372.

6. Daley GQ, et al. COVID highlights another crisis: lack of Black physicians and scientists. $\operatorname{Med}(N$ Y). 2021;2(1):2-3.

7. AlShebli BK, et al. The preeminence of ethnic diversity in scientific collaboration. Nat Commun. 2018;9(1):5163. 